\title{
photon-conserving nonlinear Schrödinger equation
}

We propose an original, simple, and direct method to measure self-steepening (SS) in nonlinear waveguides. Our proposal is based on results derived from the recently introduced photon-conserving nonlinear Schrödinger equation (NLSE) and relies on the time shift experienced by solitonlike pulses due to SS upon propagation. In particular, a direct measurement of this time shift allows for a precise estimation of the SS parameter. Furthermore, we show that such an approach cannot be tackled by resorting to the NLSE. The proposed method is validated through numerical simulations, in excellent agreement with the analytical model, and results are presented for relevant spectral regions in the near infrared, the telecommunication band, and the mid infrared, and for realistic parameters of available laser sources and waveguides. Finally, we demonstrate the robustness of the proposed scheme against deviations expected in real-life experimental conditions, such as pulse shape, pulse peak power, pulsewidth, and/or higher-order linear and nonlinear dispersion. (c) 2020 Optical Society of America

https://doi.org/10.1364/OL.401096

Self-steepening (SS) is a nonlinear effect responsible for the optical shock of ultrashort pulses that acquires singular relevance when analyzing the dynamics of broadband spectra, such as in the case of supercontinuum generation [1,2], and with applications to optical front-induced transitions [3]. SS is customarily introduced in pulse propagation models through a first-order approximation of the frequency dependence of the medium nonlinear coefficient. Known also as the "shock term," $\tau_{\mathrm{sh}}$, its relevance in the context of the modeling of supercontinuum generation was noted by Kibler, Dudley, and Coen [4]. As explained in this work (see also [5,6]), this shock term or SS parameter can be written as

$$
\tau_{\mathrm{sh}}=\frac{1}{\omega_{0}}+\frac{1}{n_{2}} \frac{d n_{2}}{d \omega}-\frac{1}{n_{\mathrm{eff}}} \frac{d n_{\mathrm{eff}}}{d \omega}-\frac{1}{A_{\mathrm{eff}}} \frac{d A_{\mathrm{eff}}}{d \omega},
$$

where the derivatives are evaluated at $\omega=\omega_{0}, n_{2}$ is the nonlinear refractive index, $n_{\text {eff }}$ is the effective refractive index, and $A_{\text {eff }}$ is the effective mode area. In a first-order approximation, the nonlinear coefficient of the waveguide is related to the SS parameter by $\gamma(\Omega)=\gamma_{0}\left(1+\tau_{\mathrm{sh}} \Omega\right)$, where $\Omega$ is the frequency detuning with respect to a conveniently chosen reference frequency $\omega_{0}$, and the SS parameter is usually given by $\tau_{\mathrm{sh}}=\omega_{0}^{-1}$, an approximation that will become clearer in what follows.

In spite of its relevance, and to the extent of our knowledge, there is not much work in the literature on the direct measurement of the SS parameter. Indeed, most of the work has focused on either the numerical estimation or the measurement of the mode effective area, rather than on the direct measurement of $\tau_{\mathrm{sh}}$. There are several reasons for this focus of literature on $A_{\text {eff. }}$ As Kibler and colleagues note [4], the nonlinear refractive index $n_{2}$ is approximately constant in many relevant materials. Indeed, the frequency dependence of $n_{2}$ is negligible far from ultraviolet resonances in fused silica [7]. We must remark, however, that this observation does not hold, for instance, in the case of plasmonic materials that incorporate metal nanoparticles (MNPs), as waveguides doped with MNPs may exhibit a zerononlinearity wavelength (ZNW), giving rise to interesting new phenomena [8,9]. Moreover, the frequency dependence of the effective mode index $n_{\text {eff }}$ is usually neglected as, in general, it is less relevant than that of the effective area.

Oftentimes, the estimation of the effective mode area dispersion is based on extensive numerical simulations or involved analytical calculations (see, e.g., [4,10-16]). Nonetheless, the mode area can also be measured (see, e.g., [17-19]). A typical experimental procedure measures the spot size, either by registering the transverse mode with a camera or by some other method (see, e.g., [20]), and the effective mode area is calculated by fitting a Gaussian distribution [5,10,21], although such a fit is not valid in general [22].

Let us emphasize once more that the SS parameter is determined not only by the frequency dependence of the mode effective area, but also by the frequency dependence of the nonlinear refractive index. An interesting example of this type 
of dependence is found in the work of Panoiu, Liu, and Osgood on silicon photonic nanowires $[23,24]$. These authors show that $\tau_{\mathrm{sh}}$ can be more than 20 times greater than $\omega_{0}^{-1}$ for some wavelengths. Moreover, they prove that the frequency dependence of the third-order susceptibility leads to significant changes in the SS parameter, even to the extent of making $\tau_{\mathrm{sh}}<\omega_{0}^{-1}$.

Besides the lack of experimental schemes allowing for the direct measurement of the SS parameter, a problem arises with the modeling of its influence. Propagation of light pulses in waveguides is usually modeled by the nonlinear Schrödinger equation (NLSE) [5]

$$
\partial_{z} \tilde{A}=i \beta(\Omega) \tilde{A}+i \gamma(\Omega) \mathcal{F}\left\{|A|^{2} A\right\},
$$

where $A=A(z, t)$ is the complex envelope of the electric field in the time domain, normalized such that $|A|^{2}$ is the optical power, and $\tilde{A}=\tilde{A}(z, \Omega)=\mathcal{F}[A(z, t)]$ where $\mathcal{F}$ stands for the Fourier transform. Coefficients $\beta(\Omega)$ and $\gamma(\Omega)$ are the linear and nonlinear dispersion profiles, respectively, and it is customary to express these profiles as Taylor expansions. It is worth noting that although the NLSE has proved to be adequate to model pulse propagation in a wide variety of cases, it is well known that it does not necessarily conserve some basic physical quantities such as the number of photons and the energy $[6,25,26]$. In particular, the photon number is preserved only if $\tau_{\mathrm{sh}}=\omega_{0}^{-1}$, a fact often overlooked in the literature, which poses a severe limitation when applying the NLSE to arbitrary nonlinear profiles $\gamma(\Omega)$. Let us define $s$ such that $\tau_{\text {sh }}=s \omega_{0}^{-1}$, i.e., $s$ is a measure of the deviation from the photon-conserving situation in the NLSE.

In order to overcome the aforementioned limitation of the NLSE, we have recently introduced a modified NLSE, the photon-conserving NLSE (pcNLSE) [27], that preserves both the energy and the number of photons in lossless waveguides. The pcNLSE reads

$\partial_{z} \tilde{A}=i \beta(\Omega) \tilde{A}+i \frac{\omega r(\Omega)}{2} \mathcal{F}\left\{C^{*} B^{2}\right\}+i \frac{\omega r^{*}(\Omega)}{2} \mathcal{F}\left\{B^{*} C^{2}\right\}$,

where $r(\Omega)=\sqrt[4]{\frac{\gamma(\Omega)}{\omega}}, \tilde{B}=r(\Omega) \tilde{A}$, and $\tilde{C}=r^{*}(\Omega) \tilde{A}$.

It can be easily verified that the pcNLSE reduces to the NLSE when $\tau_{\text {sh }}=\omega_{0}^{-1}(s=1)$, i.e., in the only case where the NLSE preserves physical quantities. For all other values of the SS parameter, however, the pcNLSE predicts different results. For instance, Fig. 1 shows results of the propagation of a short pulse, in a waveguide with $s=-1$, modeled with the pcNLSE (solid line) and the NLSE (dashed line). Not only is the predicted evolution markedly different, but also, the NLSE predicts an unphysical increase of the number of photons upon propagation, as shown in the bottom panel.

It is interesting to revisit work dealing with the influence of SS on soliton propagation in fibers [28,29]. It is found that the soliton experiences a time shift due to SS but, most remarkably, no shock occurs (in agreement with [5]). Based on this resilience of a soliton to SS, one may ponder whether such a unique feature could be used to measure the SS parameter itself. Since the NLSE will conserve the photon number only when $s=1$, we turn to explore the effect of SS on soliton propagation under the much less restrictive context of the pcNLSE, keeping in mind that by "soliton," we are referring to the fundamental soliton solution of the NLSE with no SS.
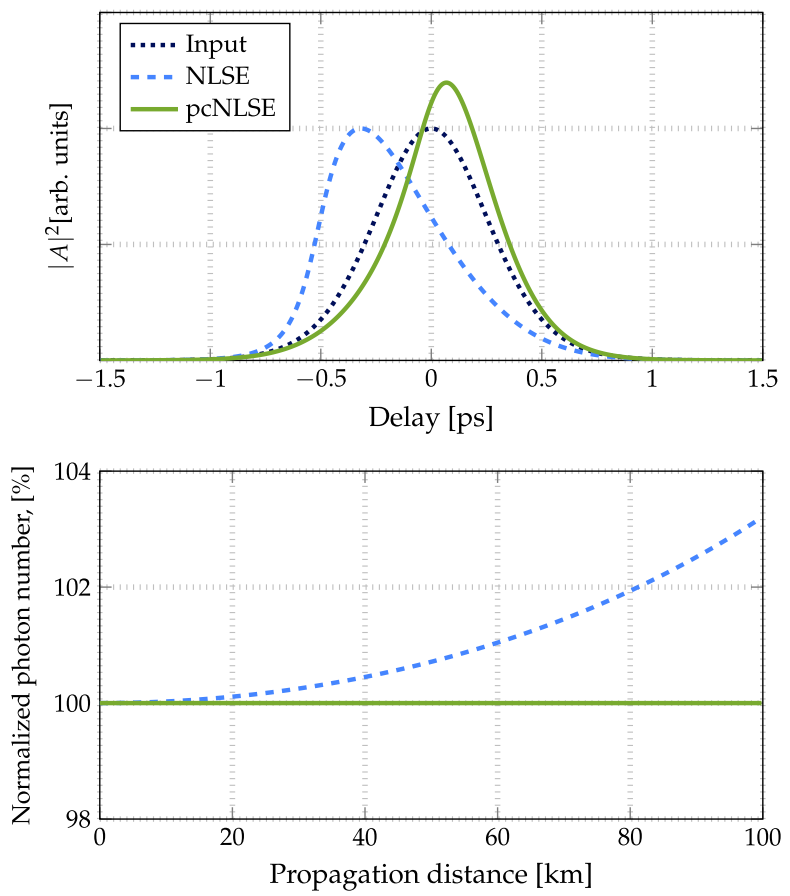

Fig. 1. Effect of self-steepening on the propagation of a short pulse in a waveguide with $s=-1$. Results with the NLSE (dashed line) and the pcNLSE (solid line). The pcNLSE preserves the photon number while the NLSE does not (bottom panel).

Results obtained with the pcNLSE are shown in Fig. 2 for different values of $s$, where we observe that not only does the soliton preserve its shape, but it is time shifted depending upon the value of the $S S$ parameter. For clearness, the time shift $\Delta_{T}$ is normalized to the pulse $1 / e$ half-width, $T_{0}$.

In order to find an analytical expression for the time shift, we must proceed in two steps. First, an approximation of Eq. (3) for narrowband pulses is developed and written in the time domain. Second, the method of moments [30-32] is applied assuming a hyperbolic secant pulse. As a result, it can be shown that (see Supplement 1 for details)

$$
\Delta_{T}(z)=\frac{s+2}{3} \frac{\gamma_{0} P_{0} z}{\omega_{0}}=\frac{s+2}{3} \frac{\beta_{2} z}{\omega_{0} T_{0}^{2}},
$$

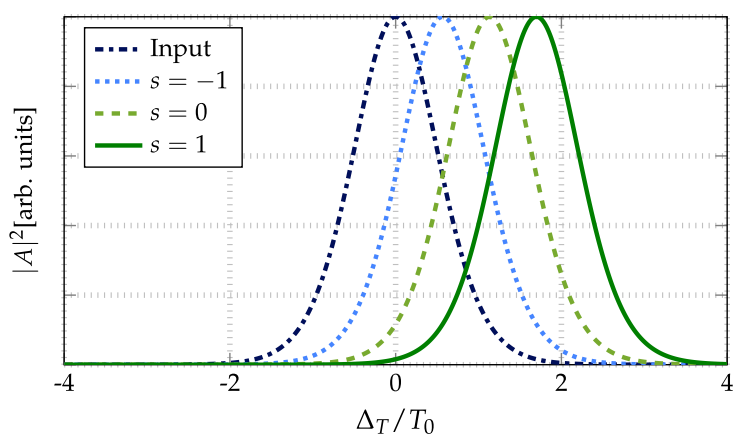

Fig. 2. Time shift experienced by a soliton due to self-steepening as predicted by the pcNLSE, and for different SS parameters: $s=-1$ (dotted line), $s=0$ (dashed line), $s=1$ (solid line); input pulse (dashed-dotted line). The time shift $\Delta_{T}$ is normalized to $T_{0}=100 \mathrm{fs}$. 


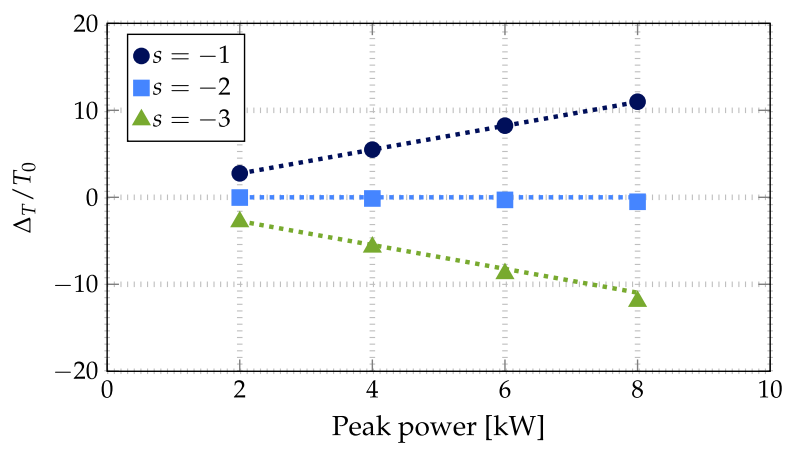

Fig. 3. Time shift experienced by a soliton, at $\lambda=1550 \mathrm{~nm}$, versus peak power and for different SS parameters: $s=-1$ (circles), $s=-2$ (squares), and $s=-3$ (triangles). Results from Eq. (4) are shown in dotted lines. Since $T_{0}=100 \mathrm{fs}, \Delta_{T} \approx \pm 1$ ps for $s= \pm 1$ and a peak power of $8 \mathrm{~kW}$.

where $\gamma_{0}$ is the zeroth-order nonlinear coefficient, $\beta_{2}$ is the group velocity dispersion, and we have neglected higher-order dispersion. This equation is valid as long as the pulse remains unchirped along propagation, a condition that was verified, by means of extensive numerical simulations, when the input was a fundamental soliton for the NLSE, i.e., $\gamma_{0} P_{0} T_{0}^{2} /\left|\beta_{2}\right|=1$.

Equation (4) suggests a direct and simple way to estimate the SS parameter, based on measuring the delay experienced by a soliton upon propagation in a nonlinear waveguide. Note also that this expression is in agreement with the delay obtained with the NLSE and from self-phase modulation (SPM) considerations, and for $s=1$, given by $\Delta_{T} \approx \gamma_{0} P_{0} z / \omega_{0}=\phi_{\max } / \omega_{0}$, where $\phi_{\max }$ is the maximum phase induced by SPM [5]. Although the delay estimated with the NLSE can be generalized for an arbitrary $s$ as $\Delta_{T} \approx s \gamma_{0} P_{0} z / \omega_{0}$, only the case of $s=1$ corresponds to a physically sound photon-conserving situation, and thus the derived SS parameter will not be reliable for any other value of $s$.

Following this line of thought, in Fig. 3, we show simulation results on the propagation of solitons with peak powers ranging from 2 to $8 \mathrm{~kW}, T_{0}=100 \mathrm{fs}$, and central wavelength $\lambda=1550 \mathrm{~nm}$ (all parameters entirely consistent with those of a femtosecond fiber laser) along a $500 \mathrm{~m}$ long fiber with $\beta_{2}=-20 \mathrm{ps}^{2} \mathrm{~km}^{-1}$ and $\gamma_{0}=1 \mathrm{~W}^{-1} \mathrm{~km}^{-1}$, both coefficients typical of a standard single-mode fiber at $1550 \mathrm{~nm}$. Also shown in the figure is the linear dependence of the time shift as obtained from Eq. (4). As we can see, there is an excellent agreement between numerical simulations and results obtained with the pcNLSE.

In a practical experimental setup, one may envision a scheme where the time shift $\Delta_{T}$ is measured by launching two pulses into the waveguide: a large amplitude pulse that is time shifted due to SS and a small amplitude pulse that is not, and thus provides a convenient reference. The delay between pulses can then be measured by techniques such as GRENOUILLE [33] and/or modern devices based on two-photon absorption detectors [34].

It is interesting to compare results on the time shift obtained with the pcNLSE with those from the NLSE. This is shown in Fig. 4, where the case of $s=-1$ from Fig. 3 is compared to its NLSE counterpart. The NLSE predicts a time shift of significantly different magnitude than that obtained with the pcNLSE and of opposite sign. This highlights the necessity of resorting to the latter equation in order to have a reliable estimate of the SS

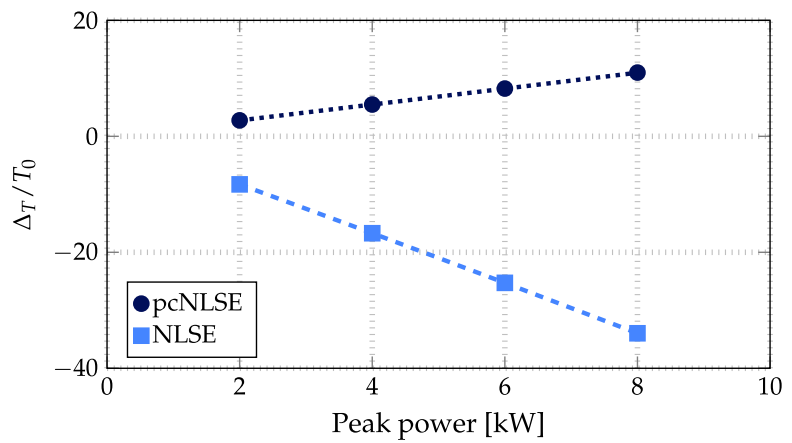

Fig. 4. Time shift versus soliton peak power for $s=-1$ and same simulation parameters as in Fig. 3. (Circles) pcNLSE and (squares) NLSE. Results from Eq. (4) for the pcNLSE are shown in dotted line. The dashed line (NLSE) is a guide to the eye. Since $T_{0}=100 \mathrm{fs}$, $\Delta_{T} \approx 1$ ps for a peak power of $8 \mathrm{~kW}$, in the case of the pcNLSE.

parameter. Note that when $s=1$, both equations will yield the same results, as this is the only case where the NLSE preserves the photon number.

Next, we are interested in validating our proposal in different spectral bands of particular relevance, namely, the near-infrared (NIR) and mid-infrared (MIR) bands. In Fig. 5, we show results of the propagation of femtosecond pulses at $\lambda=800 \mathrm{~nm}$ (top) and $\lambda=2400 \mathrm{~nm}$ (bottom), in both cases along a $10 \mathrm{~m}$ long fiber with a negative SS slope $s=-1$. In the NIR, the chosen fiber parameters are those typical of a photonic-crystal fiber (PCF) with $D=40 \mathrm{ps} \mathrm{nm}^{-1} \mathrm{~km}^{-1}$ and $\gamma_{0}=95 \mathrm{~W}^{-1} \mathrm{~km}^{-1}$; in the MIR, the chosen parameters are those of a typical ZBLAN fiber with $D=10 \mathrm{ps} \mathrm{nm}^{-1} \mathrm{k} \mathrm{m}^{-1}$ and $\gamma_{0}=1 \mathrm{~W}^{-1} \mathrm{~km}^{-1}$ [35]. As it can be readily observed, Eq. (4) makes correct predictions in both cases.

We also explore the robustness of the proposed method against deviations expected in real-life experimental conditions, such as pulse shape, peak power, and pulsewidth. In the top panel of Fig. 5, we show results obtained when considering a Gaussian-shaped pulse instead of a sech. We observe a powerdependent departure from the time shift as given by Eq. (4), but as the linear trend holds, it still allows for an estimation of the SS parameter. In the bottom panel of Fig. 5, we show results when considering deviations from the fundamental soliton condition, $N=1$; in practical terms, these could be due to either peak-power and/or pulsewidth uncertainties in an experimental setup. As apparent from the figure, results are still in excellent agreement with the model, thus supporting the applicability of the proposed method.

It is worthwhile pointing out that there could be intrinsic sources of deviations arising from effects such as waveguide losses, higher-order linear $\left(\beta_{3}\right)$ and nonlinear $\left(\gamma_{2}\right)$ dispersion, and/or the Raman-induced soliton self-frequency shift (SSFS) [5]. The effect of loss can be neglected by using a waveguide shorter than its corresponding effective length. The additional delays produced by $\beta_{3}$ and/or SSFS have been calculated analytically by using the NLSE [5,32] and these results can be shown to apply to the pcNLSE as well. As such, these contributions can be subtracted in a straightforward fashion from the total time delay, leaving only the SS contribution needed to obtain the SS parameter.

Finally, to assess the applicability of the proposed method to more general (higher-order) nonlinear profiles, Fig. 6 shows 

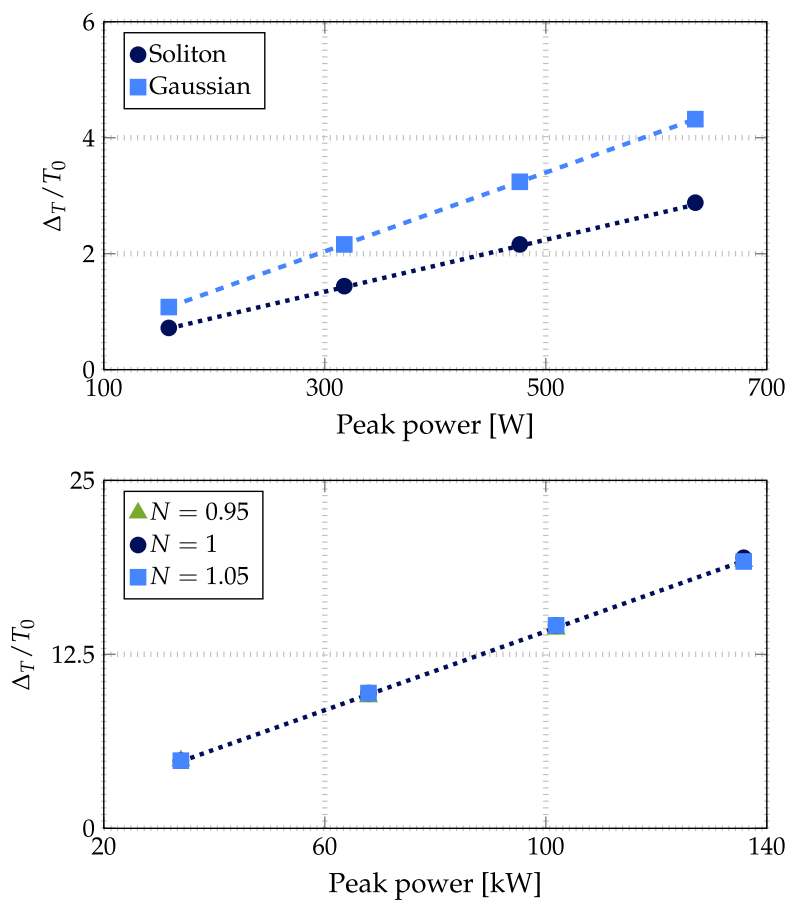

Fig. 5. Time shift versus peak power with $s=-1$ as modeled with the pcNLSE in the NIR (top) and the MIR (bottom). Results from Eq. (4) are also shown (dotted lines). Effect of a Gaussian pulse shape (top, the dashed line is a guide to the eye). (Bottom) Effect of the deviation from the fundamental soliton condition $N=1$. Since $T_{0}=30 \mathrm{fs}, \Delta_{T} \approx 0.5 \mathrm{ps}$ for a peak power of $130 \mathrm{~kW}$, in the MIR case.
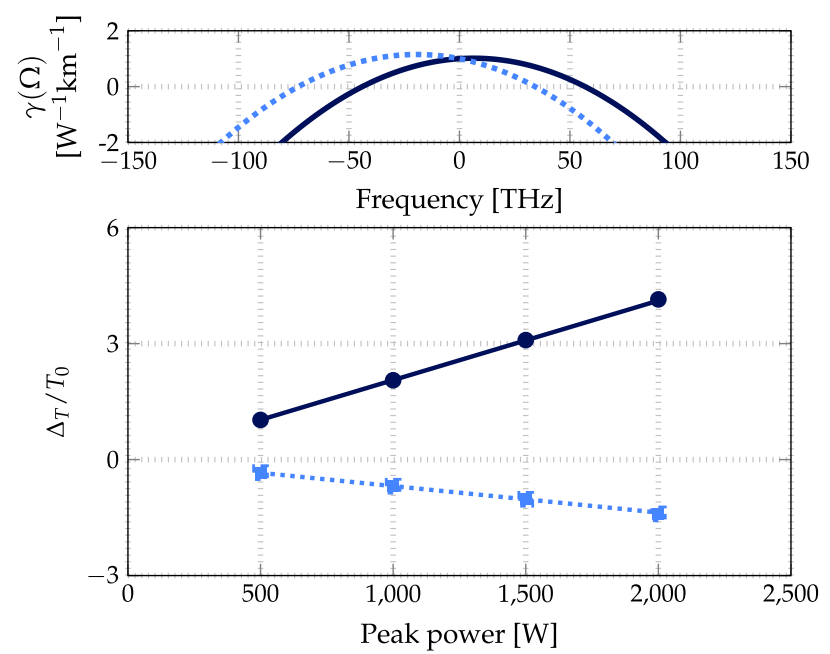

Fig. 6. (Top) Nonlinear profiles. (Bottom) Time shift experienced by a soliton, at $\lambda=1550 \mathrm{~nm}$, versus peak power in a fiber with $\gamma_{2}, s=$ 1 (solid line) and $s=-3$ (dotted line). $T_{0}=200 \mathrm{fs}$ at $500 \mathrm{~W}$. method to measure SS in nonlinear waveguides based on results derived from the recently introduced pcNLSE. Numerical results, in excellent agreement with the analytical model, were presented for relevant spectral regions in the NIR, MIR, and telecommunication bands. Finally, we showed the robustness of the proposed method against deviations expected in real-life experimental conditions, such as pulse peak power, shape, and width.

Disclosures. The authors declare no conflicts of interest.

See Supplement 1 for supporting content.

\section{REFERENCES}

1. L. Zhang, Y. Yan, Y. Yue, Q. Lin, O. Painter, R. G. Beausoleil, and A. E. Willner, Opt. Express 19,11584 (2011).

2. B. Barviau, B. Kibler, and A. Picozzi, Phys. Rev. A 79, 063840 (2009).

3. M. A. Gaafar, T. Baba, M. Eich, and A. Y. Petrov, Nat. Photonics 13 , 737 (2019).

4. B. Kibler, J. Dudley, and S. Coen, Appl. Phys. B 81, 337 (2005).

5. G. P. Agrawal, Nonlinear Science at the Dawn of the 21st Century (Springer, 2000), pp. 195-211.

6. K. J. Blow and D. Wood, IEEE J. Quantum Electron. 25, 2665 (1989).

7. D. Milam, Appl. Opt. 37, 546 (1998).

8. N. Linale, J. Bonetti, A. Sánchez, S. Hernandez, P. Fierens, and D. Grosz, Opt. Lett. 45, 2498 (2020).

9. F. Arteaga-Sierra, A. Antikainen, and G. P. Agrawal, Phys. Rev. A 98, 013830 (2018).

10. T. M. Monro, D. Richardson, N. Broderick, and P. Bennett, J. Lightwave Technol. 18, 50 (2000).

11. G. Chang, T. B. Norris, and H. G. Winful, Opt. Lett. 28, 546 (2003).

12. N. A. Mortensen, Opt. Express 10, 341 (2002).

13. K. Saitoh, M. Koshiba, T. Hasegawa, and E. Sasaoka, Opt. Express 11, 843 (2003).

14. S. A. Razzak and Y. Namihira, IEEE Photon. Technol. Lett. 20, 249 (2008).

15. Y. Wang, X. Zhang, X. Ren, L. Zheng, X. Liu, and Y. Huang, Appl. Opt. 49, 292 (2010).

16. S. Chugh, A. Gulistan, S. Ghosh, and B. Rahman, Opt. Express 27, 36414 (2019)

17. W. S. Wong, X. Peng, J. M. McLaughlin, and L. Dong, Opt. Lett. 30 , $2855(2005)$

18. M. C. P. Huy, A. Baron, S. Lebrun, R. Frey, and P. Delaye, J. Opt. Soc. Am. B 27, 1886 (2010).

19. M. C. P. Huy, A. Baron, S. Lebrun, R. Frey, and P. Delaye, J. Opt. Soc. Am. B 30, 1651 (2013).

20. J. Streckert, Opt. Lett. 5, 505 (1980).

21. D. Marcuse, J. Opt. Soc. Am. A 68, 103 (1978).

22. M. Koshiba and K. Saitoh, Opt. Express 11, 1746 (2003).

23. N. C. Panoiu, X. Liu, and R. M. Osgood, in Conference on Lasers and Electro-Optics and 2008 Conference on Quantum Electronics and Laser Science (IEEE, 2008), pp. 1-2.

24. N. C. Panoiu, X. Liu, and R. M. Osgood, Jr., Opt. Lett. 34, 947 (2009).

25. A. D. Sánchez, P. I. Fierens, S. M. Hernandez, J. Bonetti, G. Brambilla, and D. F. Grosz, J. Opt. Soc. Am. B 35, 2828 (2018).

26. A. Zheltikov, Phys. Rev. A 98, 043833 (2018).

27. J. Bonetti, N. Linale, A. Sánchez, S. Hernandez, P. Fierens, and D. Grosz, J. Opt. Soc. Am. B 36, 3139 (2019).

28. K. Ohkuma, Y. H. Ichikawa, and Y. Abe, Opt. Lett. 12, 516 (1987).

29. J. De Oliveira, M. A. de Moura, J. M. Hickmann, and A. Gomes, J. Opt. Soc. Am. B 9, 2025 (1992).

30. D. Marcuse, J. Lightwave Technol. 10, 17 (1992).

31. P.-A. Bélanger and N. Bélanger, Opt. Commun. 117, 56 (1995).

32. Z. Chen, A. J. Taylor, and A. Efimov, J. Opt. Soc. Am. B 27, 1022 (2010).

33. S. Akturk, M. Kimmel, P. O’Shea, and R. Trebino, Opt. Lett. 29, 1025 (2004).

34. J. Chen, W. Xia, and M. Wang, J. Appl. Phys. 121, 223103 (2017).

35. D. Klimentov, N. Tolstik, V. Dvoyrin, V. L. Kalashnikov, and I. T. Sorokina, J. Lightwave Technol. 30, 1943 (2012). 


\section{Query}

1. AU: You did not provide funding information to OSA at the time of article submission. If this article was funded by one or more organization(s)/institutions(s), please provide the full name of that entity as provided in the Open Funder Registry (https://search.crossref.org/funding) and all pertinent grant/contract/project/award numbers. 